DOI: http://dx.doi.org/10.15517/rce.v34i2.27323

\title{
APROXIMACIÓN METODOLÓGICA PARA EVALUAR LA EFECTIVIDAD DE PROFESORES: ESTUDIO DE LOS DOCENTES DE LA CÁTEDRA XE0156 UNIVERSIDAD DE COSTA RICA
}

\author{
José Alfonso Muñoz-Alvarado ${ }^{1}$ \\ Recibido: 30/10/2016 \\ Aprobado: 26/11/2016
}

\begin{abstract}
RESUMEN
El siguiente artículo presenta un primer acercamiento para medir de forma más precisa la efectividad de un profesor en cursos de cátedra, en este caso Introducción a la Economía para no economistas. Para lograr esto se utilizó un modelo probit basado en el modelo dinámico de efectividad y talento desarrollado por Holmström (1999), además de varias variables de control. Se logró realizar un ordenamiento de los 18 profesores de la cátedra para el segundo semestre del 2015 y, además, obtener resultados interesantes de las variables de control, como el tamaño óptimo que debería tener cada grupo.
\end{abstract}

PALABRAS CLAVE: INCENTIVOS, EFICIENCIA DE LA EDUCACIÓN, ECONOMÍA DE LA INFORMACIÓN, COSTA RICA.

\section{ABSTRACT}

The following article presents a first approach to more precisely measure the effectiveness of a teacher when giving a course in lectures, specifically in this case Introduction to Economics for non-economists. To achieve this, a probit model was used based on the dynamic model of effectiveness and talent developed by Holmström (1999), in addition to several control variables. It was possible to order the 18 professors of the chair for the second half of 2015 and also to obtain interesting results of the control variables, such as the optimal size that each group should have.

KEYWORDS. INCENTIVES, EFFICIENCY OF EDUCATION, INFORMATION ECONOMICS, COSTA RICA. 


\section{INTRODUCCIÓN}

En el entorno económico ha existido un problema entre un empleador y el empleado que se centra prácticamente en que el empleador no puede verificar el esfuerzo que realiza el empleado en la tarea asignada por el empleador. Este problema es parte de la rama económica de teoría de contratos la cual fue galardonada en el 2016 al hacerse entrega del Premio Nobel de Economía a Oliver Hart y Bengt Holmström.

El problema entre empleador y empleado se puede trasladar a un entorno educativo donde un centro de enseñanza contrata a un docente; sin embargo, el problema se acentúa en este campo ya que la calidad del docente no solamente afecta al empleador, sino que su esfuerzo resulta en una mejor o peor educación de los estudiantes, lo cual puede afectar el desarrollo de una comunidad o incluso la de un país. De allí que se han diseñado distintos mecanismos para la realización de evaluaciones docentes. Parraguez y Hoffman (2011) concluyen que en España "en ausencia de eficientes mecanismos de control de la conducta tanto del profesor como del estudiante pueden proliferar vicios de responsabilidad que atentan contra la calidad del proceso de enseñanza-aprendizaje". Este resultado resalta la importancia de estudiar y conocer más sobre la calidad de la educación por medio del esfuerzo que realiza un profesor al dar un curso. Varios países han desarrollado mecanismos para obtener estimaciones de la eficiencia con la que un profesor dicta un curso. Por ejemplo, Ruíz (2012) realiza un estudio sobre la calidad docente en Venezuela utilizando las notas de los estudiantes en diferentes cursos. También se tiene el estudio en Colombia de García, Cerda y Donoso-Díaz (2011), en el que, por medio de un modelo probit, analizan la calidad docente y diferentes mecanismos para incentivar un mejor desempeño de los docentes, encontrando relaciones entre variables socio-económicas de los docentes y la probabilidad de participar y ser acreditado en el Programa de Acreditación para la Asignación de Excelencia Pedagógica de dicho país.

El caso de Costa Rica resulta de interés, dado el modelo educativo que ha seguido, con una alta participación del estado en el financiamiento de la educación en todos los niveles, incluyendo el universitario desde 1940. A pesar de que en la Universidad de Costa Rica, que es su principal institución de educación superior existe un Centro de Evaluación Académica, la evaluación a los docentes se limita fundamentalmente al cuestionario elaborado por dicho centro y que llenan voluntariamente los estudiantes de cada curso que brinda la universidad. Existen varios estudios basados en estas evaluaciones para conocer más sobre la confiabilidad y eficiencia de ese mecanismo; por ejemplo, el trabajo de Araya y Vargas (2013), el cual encontró una relación positiva entre la nota promedio de los cursos y la nota obtenida por el profesor en la evaluación docente.

Sin embargo, la literatura sobre este mecanismo a nivel nacional se centra en estadística descriptiva lo cual puede limitar los resultados obtenidos. El presente trabajo busca crear una nueva forma de medir el desempeño docente incluyendo variables de los profesores, los estudiantes y del grupo. Al incluir todas estas variables se logra ampliar los estudios de Ruiz (2012) y García et al. (2011), los cuales se limitaban a sólo uno de estos tres ámbitos.

Conocer el desempeño de un profesor al dar clases es muy complejo. Primero es muy difícil ir a todas las clases a evaluar la forma de enseñanza de cada profesor ya que su costo es muy elevado. Segundo, los instrumentos que utiliza un profesor varían debido a la diferencia entre las características que puedan existir entre los docentes y entre los grupos: cantidad de alumnos, años de experiencia, tipo de alumnos, curso que enseña y demás. Sin embargo, existe la necesidad de conocer la efectividad de cada profesor en su clase con el fin de mantener, cambiar o capacitar a dicho profesor según las necesidades de los estudiantes y de cada unidad académica. Este nuevo mecanismo también se podría ampliar para utilizarse en otros cursos universitarios con facilidad, ya que la mayoría de variables utilizadas en el presente modelo se pueden obtener fácilmente de las bases de datos institucionales y por tanto mejorar la evaluación de profesores universitarios y la formación de los estudiantes. El presente 
estudio permitiría, además, abrir nuevas oportunidades de investigación al unir teoría de contratos y teoría de la educación a nivel nacional, cosa que no se había hecho antes en Costa Rica.

Para tratar de manejar estos problemas es idóneo trabajar en un curso de cátedra, como Introducción a la Economía para no economistas, donde los estudiantes que matriculan tengan similar conocimiento inicial de la materia, los profesores trabajen bajo un mismo programa y, más importante aún, el grueso ( $67.5 \%$ en este caso) de la nota del estudiante es definida por la cátedra y no por el profesor.

El modelo utilizado se basa en el modelo dinámico de efectividad y talento desarrollado por Holmström (1999). Por medio de un modelo probit y el uso de variables de control se obtuvo una medición de la efectividad, o desempeño, que presentó cada profesor a la hora de que los estudiantes aprobaran y luego ordenar dicha efectividad.

Es importante mencionar que las variables de control permiten eliminar las diferencias que existen entre profesores y entre los grupos de estudiantes ya que, como lo explican Milgrom \& Roberts (1992), no es lo mismo la efectividad en un profesor con buenos estudiantes y muchos años de dar el curso que uno nuevo con un grupo de estudiantes no tan buenos, aunque la cantidad de alumnos que aprueban con cada profesor sea la misma; en este caso, la efectividad del segundo profesor es mayor.

Con el modelo estimado es posible obtener una nueva medición de la efectividad de cada profesor para que sus estudiantes aprueben el curso, permitiendo crear un nuevo ranking de profesores para comparar con el ranking obtenido con las notas de la evaluación docente del Centro de Evaluación Académica.

\section{METODOLOGÍA}

En 1999 Holmström planteó un modelo en el cual se relaciona la producción de un empleado con su talento y la efectividad que realiza a lo largo de múltiples periodos. El talento definido como las cualidades propias que cada persona posee intrínsecamente; la efectividad, por otro lado, es el esfuerzo dado en la realización del trabajo que se está haciendo. Este modelo se desarrolla a partir de la siguiente ecuación:

$$
y_{t}=\eta+a_{t}+\varepsilon_{t}, t=1,2, \ldots
$$

Donde el producto es $y_{\mathrm{t}}$, el trabajo del agente es $\mathrm{a}_{\mathrm{t}}, \varepsilon_{t}$ un error estocástico i.i.d. $y$ por último, $\eta$ se define como el talento del agente y el cual no es conocido por el principal inicialmente, aunque a lo largo del tiempo se comienza a reflejar. En equilibrio se obtiene la siguiente secuencia:

$$
z_{t} \equiv \eta+\varepsilon_{t}=y_{t}-a_{t}^{*}\left(y^{\mathrm{t}-1}\right)
$$

Por medio de la observación de $z_{t}$ a lo largo del tiempo se logra conocer el talento del agente dado que $\varepsilon_{t} \rightarrow 0$ cuando $t \rightarrow \infty$. En este ensayo se asumirá la relación lineal presentada en la ecuación 1, sin embargo, Holmström (1999) hace énfasis que la relación puede ser no lineal de la forma:

$$
\begin{gathered}
y_{t}=\eta_{t} * a_{t} \\
y_{t}=f\left(\eta_{t}\right)+a_{t}
\end{gathered}
$$


Esta no linealidad podría permitir una relación donde la cantidad de producto sea según la cantidad de trabajo y el talento del agente en conjunto. Esto haría que dos trabajadores con diferentes talentos y trabajo puedan producir el mismo producto.

\section{Datos: Introducción a la Economía para no economistas}

El curso de Introducción a la Economía para no economistas en un curso de la Escuela de Economía de la Universidad de Costa Rica. Al ser un curso de servicio y repertorio permite a gente de cualquier carrera de la universidad llevarlo, aunque muchas carreras lo tienen como curso del plan de estudios.

Para el segundo semestre del 2015 el curso estuvo estructurado en 3 exámenes parciales que combinados sumaban el $90 \%$ de la nota del estudiante y un $10 \%$ que correspondía a pruebas cortas, trabajos o tareas creadas por cada profesor. Los exámenes se dividían en dos partes, una parte de selección única a cargo de la cátedra, dicha sección representaba 75 \% de la nota del examen. La segunda parte era una parte de desarrollo que representaba el $25 \%$ faltante de la nota. Esta parte de desarrollo se basaba en dos noticias de medios nacionales o internacionales común para todos los profesores, donde cada profesor tenía la libertad de preguntar lo que quisieran. Con esta estructura del curso la cátedra se aseguraba que los estudiantes tuvieran un mismo nivel mínimo de entendimiento sobre la economía al salir del curso sin perder la libertad de cátedra de cada profesor.

En el segundo semestre del 2015 el curso de Introducción a la Economía se conformó por 22 grupos en la sede Rodrigo Facio. 18 profesores estaban a cargo de dar el curso, donde 1 profesor dio 4 cursos y otro profesor dio 2 cursos, los demás profesores dieron 1 curso nada más. 779 estudiantes de diversas carreras matricularon inicialmente el curso, pero 122 realizaron retiro de matrícula por lo que la matrícula efectiva fue de 657 estudiantes. Al finalizar el curso, solo 297 estudiantes, correspondiente al 45,2 \% de la matrícula efectiva, aprobaron el curso.

\section{Aplicación}

Usando el modelo probabilístico probit y variando el modelo de Holmström (1999) para que sea de un solo periodo, $y$ por tanto sea posible utilizarlo con los datos, se obtuvo de la ecuación 1:

$$
y=\delta_{j}^{*} \eta_{j}+\theta^{*} N+\mu^{*} P+\beta_{i}^{*} X_{i}+\varepsilon
$$

Al ser el modelo de un solo periodo es posible encontrar la efectividad y el talento que tuvo cada profesor a lo largo del curso durante el segundo semestre del 2015; sin embargo, para un estudio de un solo periodo no se pueden estimar la efectividad y el talento por separado, solamente se pueden obtener de forma conjunta.

Se utiliza $\mathrm{X}_{\mathrm{i}}$ que representa un vector de variables de control que sirven para absorber las diferencias que pueden existir entre estudiantes y entre grupos. Las variables de control utilizadas se muestran en el cuadro 1. Las variables dummy de "carreras" utilizan como base las carreras de Ciencias Económicas: Administración de Negocios, Estadística, Contaduría y Aduanas. La dummy de Ciencias Sociales incluye las carreras de Sociología, Ciencias Políticas e Historia; Derecho no se incluyó por el hecho que hubo un grupo que en su mayoría los estudiantes pertenecían a dicha carrera. La variable promedio de matrícula corresponde al promedio de la nota de matrícula de los estudiantes de cada grupo. Matrícula efectiva es la resta entre la cantidad de estudiantes matriculados en el curso y los estudiantes que realizan retiro o interrupción durante el semestre. Se tienen 
otras variables de control: cantidad de estudiantes que evaluaron al profesor, cantidad de semestres que ha dado el curso y una dummy de régimen académico, sin embargo, estas variables produjeron problemas de multicolinealidad en el modelo por lo cual no se utilizaron.

CUADRO 1

VARIABLES DE CONTROL

\begin{tabular}{l}
\hline Promedio de matrícula \\
Dummy de carrera (Ciencias Económicas base) \\
Matrícula efectiva \\
(Matrícula efectiva)^ 2 \\
Variable categórica del horario (7am a 11am base) \\
\hline
\end{tabular}

Fuente: Elaboración propia.

En el modelo, $y$ es la variable dicotómica de aprobación del estudiante, donde 1 corresponde a un estudiante que aprobó el curso. Aunque la variable $\eta$ no es visible en el modelo de Holmström (1999) en este ensayo se definió como j-1 dummys que diferencian entre los j profesores, siendo la categoría base el profesor que denotamos como A, permitiendo que $\delta_{j}$ sea la efectividad no mostrada por la nota del profesor. $\theta$ es el coeficiente asociado a la variable de Nota del profesor $(\mathrm{N})$ el cual es la efectividad asignada por los estudiantes y $\mu$ es el coeficiente asociado a la variable Porcentaje de participación en la evaluación (P) la cual denota el porcentaje de estudiantes que realizó la evaluación docente del profesor y la cual permite obtener una señal de eficiencia ya que los estudiantes responden las evaluaciones bajo un sesgo positivo o negativo hacia el profesor.

Es importante recalcar que muchos estudiantes no contestan las evaluaciones de los cursos, los que lo contestan es posible que sea por mostrar una gratitud hacia el profesor al otorgarle una nota alta o más bien castigarlo otorgándole una nota mala. En este trabajo se utilizó una nota promedio ajustada por participación que obtiene la Escuela de Economía y se le asignó a cada uno de los estudiantes de cada grupo, según el profesor con el que matriculó el curso. Para los profesores que dieron el curso a más de un grupo se les asignó un promedio ponderado por grupo de dicha nota.

$\mathrm{Al}$ obtener estos tres coeficientes podemos obtener la efectividad total como:

$$
E_{T}=\delta_{j}^{*} \eta_{j}+\theta^{*} N_{j}+\mu^{*} P_{j}, \quad j=\text { profesores }
$$

\section{RESULTADOS}

En esta sección se presentan los resultados obtenidos al aplicar el modelo probit.

El modelo trabaja con errores estándar con cluster entre grupos (cuadro 2). La razón de usar este método es que es muy probable que dentro de cada grupo haya características propias y diferentes a otros grupos. Especialmente por el hecho que amigos o conocidos matriculen en cierto grupo juntos $y$ se apoyen durante el semestre.

El modelo es la versión final de una serie de modelos estimados, diferenciados según las variables de control. En las distintas corridas el coeficiente asociado a la dummy de los profesores identificados con las letras C, R, D y M no eran diferentes a la del profesor base A, por esta razón se 
eliminaron del modelo. Lo mismo pasó con las carreras de Derecho, Matemáticas, Química, Medicina y Otros y con la categoría del horario entre $3 \mathrm{pm}$ a $7 \mathrm{pm}^{2}$.

En el cuadro 2, se presentan los efectos marginales del modelo, los cuales son todos diferentes de cero al $1 \%$ de significancia.

CUADRO 2

MODELO 2: EFECTOS MARGINALES

\begin{tabular}{|c|c|c|}
\hline \multicolumn{3}{|c|}{ Errores estándar con cluster por grupos } \\
\hline Probabilidad de Aprobar & $\begin{array}{l}\text { Correctamente calcu- } \\
\text { lado:70,49 \% }\end{array}$ & Pseudo $R 2=0,2018$ \\
\hline Número de observaciones $=654$ & $\mathrm{AIC}=738,025$ & $\mathrm{BIC}=782,8561$ \\
\hline Variables & Efecto Marginal & Error Estándar \\
\hline Promedio de matrícula & 0,137 & 0,014 \\
\hline Matrícula efectiva & 0,711 & 0,041 \\
\hline Matrícula efectiva al cuadrado & $-0,012$ & 0,001 \\
\hline \multicolumn{3}{|l|}{ Horario (base: 7 a 11 y 3 a 7 ) } \\
\hline $11 \mathrm{am}$ a $3 \mathrm{pm}$ & 0,081 & 0,009 \\
\hline Nota del Profesor & 0,149 & 0,008 \\
\hline Participación en evaluación & $-0,500$ & 0,071 \\
\hline \multicolumn{3}{|l|}{ Carreras } \\
\hline Ingeniería & 0,159 & 0,047 \\
\hline Ciencias Sociales & $-0,211$ & 0,057 \\
\hline Zootecnia & $-0,316$ & 0,105 \\
\hline \multicolumn{3}{|l|}{ Profesores } \\
\hline B & 0,316 & 0,014 \\
\hline $\mathrm{E}$ & 0,289 & 0,014 \\
\hline $\mathrm{F}$ & 0,569 & 0,001 \\
\hline $\mathrm{H}$ & 0,528 & 0,001 \\
\hline G & 0,349 & 0,010 \\
\hline $\mathrm{J}$ & 0,540 & 0,001 \\
\hline K & $-0,256$ & 0,010 \\
\hline $\mathrm{L}$ & 0,188 & 0,011 \\
\hline$P$ & 0,549 & 0,001 \\
\hline $\mathrm{N}$ & 0,483 & 0,011 \\
\hline $\mathrm{O}$ & 0,548 & 0,001 \\
\hline Q & 0,539 & 0,001 \\
\hline
\end{tabular}

Fuente: Elaboración propia.

2 Si desea conocer más sobre los demás modelos y sus resultados puede solicitar los diferentes comandos en Stata al autor. 
Basándose en el modelo y usando la ecuación 6 se puede ordenar el efecto marginal de cada profesor y obtener el cuadro 3. Dicho cuadro fue ordenado de mayor a menor con el fin de obtener las mayores efectividades entre los profesores y así lograr identificar el profesor que presentó la mayor efectividad, dado todas las variables de control que se utilizaron. Se presenta también la nota de la evaluación docente, el porcentaje de estudiantes respecto al total de estudiantes de cada grupo que participaron en dicha evaluación, el efecto marginal obtenido en el modelo, la efectividad total y el ranking por nota con el fin de presentar un estudio más completo y poder emparejar diferencias entre ambos rankings.

CUADRO 3

RANKING DE LA EFICIENCIA DE PROFESORES

\begin{tabular}{lccccc}
\hline Profesor & Efectividad & Nota & $\begin{array}{c}\text { Porcentaje } \\
\text { Evaluación }\end{array}$ & $\begin{array}{c}\text { Porcentaje de } \\
\text { aprobados }\end{array}$ & $\begin{array}{c}\text { Promedio de } \\
\text { Matrícula }\end{array}$ \\
\hline F & 1,833 & 9,740 & $37,500 \%$ & $62,500 \%$ & 8,566 \\
O & 1,770 & 9,250 & $31,300 \%$ & $47,100 \%$ & 6,802 \\
P & 1,680 & 9,420 & $54,500 \%$ & $50,000 \%$ & 6,782 \\
H & 1,672 & 9,650 & $58,800 \%$ & $85,294 \%$ & 8,079 \\
J & 1,659 & 9,100 & $47,400 \%$ & $36,842 \%$ & 6,758 \\
N & 1,631 & 9,030 & $39,500 \%$ & $65,789 \%$ & 7,523 \\
Q & 1,447 & 7,980 & $56,300 \%$ & $31,250 \%$ & 6,054 \\
B & 1,422 & 9,330 & $56,800 \%$ & $48,649 \%$ & 8,075 \\
E & 1,401 & 9,500 & $60,600 \%$ & $63,636 \%$ & 6,878 \\
L & 1,369 & 9,270 & $40,000 \%$ & $54,286 \%$ & 6,893 \\
G & 1,337 & 8,480 & $55,000 \%$ & $20,000 \%$ & 7,161 \\
D & 1,297 & 9,640 & $27,800 \%$ & $25,000 \%$ & 6,749 \\
R & 1,155 & 9,250 & $44,663 \%$ & $61,538 \%$ & 7,579 \\
A & 1,146 & 9,060 & $40,700 \%$ & $35,700 \%$ & 7,934 \\
I & 1,136 & 9,360 & $51,700 \%$ & $41,379 \%$ & 6,851 \\
C & 1,069 & 8,570 & $41,657 \%$ & $30,583 \%$ & 7,412 \\
M & 1,023 & 8,100 & $36,700 \%$ & $13,333 \%$ & 5,725 \\
K & 0,863 & 9,040 & $45,500 \%$ & $24,242 \%$ & 8,028 \\
\hline
\end{tabular}

Fuente: Elaboración propia.

En el cuadro anterior se puede observar la nota obtenida por cada profesor de los estudiantes y el ranking estimado por el modelo. En la figura 1 se puede apreciar la relación positiva entre ambas variables. 
GRÁFICO 1

NOTA VS EFECTIVIDAD

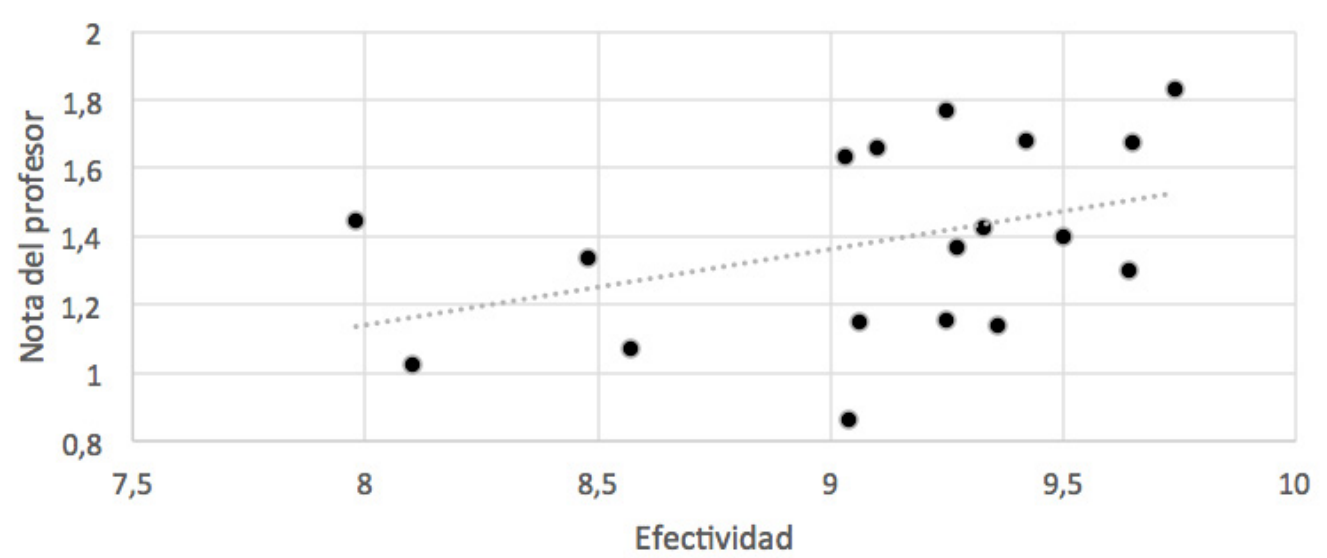

Fuente: Elaboración propia.

Existen muchas diferencias en la posición de la mayoría de profesores, aunque algunos mantuvieron la posición similar en ambos rankings. La diferencia en posición en el ranking obtenido por el modelo se puede deber a varias razones: primero, al utilizar variables de control se descartan los efectos externos a la efectividad del profesor. Por ejemplo, no es lo mismo dar clase a un grupo de estudiantes malos que a uno bueno, el modelo al utilizar como variable de control el promedio de matrícula del estudiante toma en cuenta la diferencia que existe en el desempeño de los estudiantes de cada grupo, algo que la nota del profesor obtenida en la evaluación no hace.. Segundo, el ranking por nota presenta dos problemas muy importantes: no todos los estudiantes responden dicho cuestionario y muchos estudiantes pueden tener un sesgo positivo o negativo hacia el profesor, razón por la cual podrían tener un mayor interés en contestar el cuestionario. En el modelo se obtiene un signo negativo en el coeficiente asociado a la variable de la participación estudiantil en la evaluación docente, lo cual muestra un sesgo negativo de estudiantes hacia el profesor, por lo que en promedio se puede decir que los estudiantes contestan la evaluación con el fin de mostrar su disconformidad.

Complementando estos resultados, se obtuvo lo siguiente: primero el hecho que la carrera del estudiante y el promedio de matrícula juegan un papel importante a la hora de aprobar el curso, siendo Zootecnia y Ciencias Sociales las carreras donde los estudiantes disminuyen su probabilidad de aprobar.

Por otro lado, el horario en que se da el curso juega un papel pequeño pero significativo para que los estudiantes aprueben el curso, donde los grupos de 11am a 3pm presentan una mayor probabilidad de aprobación. Al ver este resultado se podría argumentar que la variable de horario funciona como un proxy de la dedicación que podría tener un estudiante a los estudios, dado que es muy improbable que alguien que trabaje lleve un curso entre las 1lam y las 3pm.

Por último, utilizando los coeficientes de matrícula efectiva y su cuadrado se obtiene la siguiente ecuación:

$$
\text { Matrícula }=0,711^{*} \text { Matrícula }-0,012 \text { Matrícula }^{2}
$$

Al optimizar la ecuación anterior se obtiene Matrícula = 29,62; es decir, el tamaño óptimo de un grupo es de 30 estudiantes. Este último resultado puede influir significativamente en los estudiantes y en la efectividad que puede presentar un profesor. 


\section{CONCLUSIONES}

Se concluye que es posible crear un nuevo mecanismo por el cual se puede evaluar la efectividad con la que un profesor dicta un curso, contemplando dentro del mismo características propias del profesor, el estudiante y el curso impartido. Además, complementando este resultado se obtuvo que es posible estimar una cantidad óptima de estudiantes por curso y un horario que influye en la nota del estudiante y en la efectividad del profesor.

Con el cuadro 3 se obtuvo el ranking para encontrar el profesor con la mayor efectividad, el cual sería el profesor identificado con la letra F; aunque dicho profesor también ocupa el primer lugar en el ranking creado por la nota de la evaluación docente, no resulta así para los demás profesores. No obstante, es necesario considerar ciertas particularidades del curso y buscar la forma de manejarlas. Por ejemplo, muchos estudiantes, aunque matriculen con un profesor atienden a clases de otros profesores o nada más se presentan para los exámenes, podría existir diferencia en esfuerzo del estudiante si matricularon por inclusión o no ya que el tiempo de clases difiere. También es importante mencionar y tratar de obtener datos de variables que podrían resolver los problemas de estimación, por ejemplo, la cantidad de clases que da el profesor por semestre puede diferir significativamente entre varios profesores según su salud o agenda profesional de viajes internacionales y las veces que el estudiante ha repetido el curso. Este ejemplo resulta en otro problema que podría surgir y es la sustitución en ciertas clases de un profesor por otro por motivos de ausencias, esto genera que ciertos estudiantes, aunque sea por un corto periodo, reciban dos efectos de profesores diferentes y por tanto afecte su rendimiento académico. Por otra parte, la linealidad del modelo de Holmström (1999) puede no ser la mejor especificación a utilizar en docencia ya que la experiencia del profesor puede influir bastante dentro del esfuerzo para hacer que los estudiantes entiendan conceptos, además de tenerse una mayor gama de ejemplos adquirida a lo largo de los años.

Aunque el trabajo tiene limitaciones, el ranking obtenido es un primer paso para mejorar la estimación de la efectividad o el esfuerzo de los profesores, mostrando resultados más completos que los obtenidos en estudios anteriores por Ruíz (2012) y García et al. (2011). Es recomendable que la investigación se extienda a semestres adicionales y trabajar con un panel, lo cual permitiría estimar el talento, el cual es fijo para los profesores, y la efectividad del profesor, la cual varía por semestres. El trabajo abre la puerta a una nueva forma de evaluación docente más eficiente y precisa posibilitando más estudios que permitan combinar teoría de contratos y economía de la educación. También es posible extender el estudio a otros cursos de la Universidad de Costa Rica, lo que llevaría a mejorar la recolección de datos para la evaluación de los docentes, con el fin de proveer criterios para programas de capacitación orientados a mejorar su efectividad con el consecuente beneficio para los estudiantes.

\section{AGRADECIMIENTOS}

Estoy profundamente agradecido con el profesor Miguel Cantillo por su ayuda tanto de forma teórica como empírica en la realización de este trabajo.

También a la Escuela de Economía por permitirme utilizar los datos de la Cátedra de Introducción a la Economía para realizar este estudio. 


\section{REFERENCIAS}

Araya, C., \& Vargas, É. (enero-abril, 2013). Evaluación docente. ¿Mecanismo equitativo y confiable o proceso viciado que no cumple con su objetivo?. Revista Electrónica "Actualidades Investigativas en Educación”, 13(1), 1-31. http://dx.doi.org/10.15517/aie.v13i1.11709

García, L. Y., Cerda, A. A., \& Donoso-Díaz, S. (enero-marzo, 2011). Determinantes de la calidad docente: Programa de Asignación de Excelencia Pedagógica. Innovar, 21(39), 7-22. Recuperado de http://www.fce.unal.edu.co/media/files/documentos/Innovar/v21n39/v21n39.pdf

Holmström, B. (1999). Managerial Incentive Problems: A Dynamic Perspective. Review of Economic Studies, 66(1), 169-182. http://dx.doi.org/10.1111/1467-937X.00083

Milgrom, P. \& Roberts, J. (1992) Economics, Organization and Management, Pearson.

Ruíz, G. (noviembre, 2012). Rendimiento académico y evaluación docente. Revista Varianza, 9, 24-30.

Parraguez, J., \& Hoffmann, S. (2011). El proceso de enseñanza-aprendizaje universitario: reflexiones a través de la teoría de agencia. Teoría de la Educación. Educación y Cultura en la Sociedad de la Información, 12(1), 27. Recuperado de http://revistas.usal.es/index.php/revistatesi/ article/view/7836 